\title{
ON NONBINARY 3-CONNECTED MATROIDS
}

\author{
JAMES G. OXLEY
}

\begin{abstract}
It is well known that a matroid is binary if and only if it has no minor isomorphic to $U_{2,4}$, the 4-point line. Extending this result, Bixby proved that every element in a nonbinary connected matroid is in a $U_{2,4^{-}}$ minor. The result was further extended by Seymour who showed that every pair of elements in a nonbinary 3 -connected matroid is in a $U_{2,4}$-minor. This paper extends Seymour's theorem by proving that if $\{x, y, z\}$ is contained in a nonbinary 3-connected matroid $M$, then either $M$ has a $U_{2,4}$-minor using $\{x, y, z\}$, or $M$ has a minor isomorphic to the rank-3 whirl that uses $\{x, y, z\}$ as its rim or its spokes.
\end{abstract}

1. Introduction. This paper proves a number of results on the structure of nonbinary 3-connected matroids. In [8], Seymour showed that if $M$ is such a matroid and $\{x, y\} \subseteq E(M)$, then $M$ has a $U_{2,4}$-minor that uses $\{x, y\}$, that is, $M$ has a minor isomorphic to $U_{2,4}$ whose ground set contains $\{x, y\}$. Seymour also conjectured $[\mathbf{8}, \mathbf{1 0}]$ that if $M$ is nonbinary and 4-connected and $\{x, y, z\} \subseteq$ $E(M)$, then $M$ has a $U_{2,4}$-minor using $\{x, y, z\}$. In addition, he noted that this conjecture fails for certain 3-connected matroids such as the non-Fano matroid. This paper does not attack Seymour's conjecture directly but instead concentrates on characterizing precisely when the conjecture fails for nonbinary 3-connected matroids. The author has learned, since the original submission of this paper, that Seymour's conjecture has now been disproved by Kahn [4].

Most of the matroid terminology used here will follow Welsh $[\mathbf{1 2}]$. The ground set, rank, and corank of the matroid $M$ will be denoted by $E(M)$, rk $M$, and cork $M$ respectively. For an arbitrary subset $T$ of $E(M), \operatorname{rk} T$ and cork $T$ will denote the rank and corank of $T$. The deletion of $T$ from $M$ will be denoted by $M \backslash T$ or $M \mid(E(M)-T)$, and the contraction of $T$ from $M$ by $M / T$ or $M \cdot(E(M)-T)$. Flats of $M$ of ranks one and two will be called points and lines. A 3-element circuit of $M$ will be called a triangle and a 3-element cocircuit a triad.

If $N$ and $M$ are matroids on $S$ and $S \cup e$ respectively and $e \notin S$, then $M$ is an extension of $N$ if $M \backslash e=N$, and $M$ is a lift of $N$ if $M^{*}$ is an extension of $N^{*}$. We call $M$ a nontrivial extension of $N$ if $e$ is neither a loop nor a coloop of $M$ and $e$ is not in a 2-element circuit of $M$. Likewise, $M$ is a nontrivial lift of $N$ if $M^{*}$ is a nontrivial extension of $N^{*}$.

Received by the editors August 2, 1984. Presented at the Special Session on the Structure of Graphs and Matroids at the American Mathematical Society Summer Meeting in Eugene, Oregon, August, 1984.

1980 Mathematics Subject Classification (1985 Revision). Primary 05B35.

This research was partially supported by an LSU Faculty Summer Research Grant. 
If $k$ is a positive integer, the matroid $M$ is $k$-separated if there is a subset $T$ of $E(M)$ such that $|T| \geq k,|E(M)-T| \geq k$, and

$$
\operatorname{rk} T+\operatorname{rk}(E(M)-T)-\operatorname{rk} M=k-1 .
$$

When this occurs, $(T, E(M)-T)$ is an exact $k$-separation of $M$. We say that $M$ is $n$-connected if $M$ is not $k$-separated for any $k<n$. Thus a matroid is 2 -connected precisely when it is connected $[\mathbf{1 2}$, p. 69]. Moreover,

(1.1) $M$ is $n$-connected if and only if $M^{*}$ is $n$-connected.

We shall assume familiarity with the operations of series and parallel connection of matroids; a detailed discussion of these operations and their properties may be found in [3]. For matroids $M_{1}$ and $M_{2}$ on disjoint sets, if $p_{1} \in E\left(M_{1}\right)$ and $p_{2} \in E\left(M_{2}\right)$, then we shall denote the parallel connection of $M_{1}$ and $M_{2}$ with respect to the basepoints $p_{1}$ and $p_{2}$ by $P\left(\left(M_{1}, p_{1}\right),\left(M_{2}, p_{2}\right)\right)$ or just $P\left(M_{1}, M_{2}\right)$. If, for $i=1,2, M_{i}$ has at least 3 elements and $p_{i}$ is neither a loop nor a coloop of $M_{i}$, then $P\left(\left(M_{1}, p_{1}\right),\left(M_{2}, p_{2}\right)\right) \backslash p$ will be called the 2-sum of $M_{1}$ and $M_{2}[7$, p. 308]. In that case, $M_{1}$ and $M_{2}$ are the parts and $p_{1}$ and $p_{2}$ the basepoints of the 2 -sum. The following fundamental link between 3 -connection and 2-sums was proved by Seymour $[\mathbf{7},(2.6)]$.

(1.2) THEOREM. If $\left(X_{1}, X_{2}\right)$ is an exact 2-separation of the matroid $M$, then there are matroids $M_{1}$ and $M_{2}$ on $X_{1} \cup p_{1}$ and $X_{2} \cup p_{2}$ respectively, where $p_{1}$ and $p_{2}$ are new elements, such that $M$ is the 2-sum of $M_{1}$ and $M_{2}$ with respect to the basepoints $p_{1}$ and $p_{2}$. Conversely, if $M$ is the 2 -sum of $M_{1}$ and $M_{2}$, then $\left(E\left(M_{1}\right)-p_{1}, E\left(M_{2}\right)-p_{2}\right)$ is an exact 2-separation of $M$, and $M_{1}$ and $M_{2}$ are isomorphic to minors of $M$.

We shall need to use the construction of a minor of $M$ isomorphic to $M_{1}$. This proceeds as follows. Let $C$ be a circuit of $M$ meeting both $E\left(M_{1}\right)-p_{1}$ and $E\left(M_{2}\right)-$ $p_{2}$. Choose an element $z$ of $C$. Now delete $E\left(M_{2}\right)-p_{2}-C$ and then contract $C-z-E\left(M_{1}\right)$ from $M$. The resulting minor of $M$ is isomorphic to $M_{1}$.

The following two properties of 2-sums are straightforward to check.

(1.3) The sum of $M_{1}$ and $M_{2}$ is connected if and only if both $M_{1}$ and $M_{2}$ are connected.

(1.4) If $M$ is the 2-sum of matroids $M_{1}$ and $M_{2}$ and $N$ is a 3-connected matroid which is a minor of $M$, then $M_{1}$ or $M_{2}$ has an $N$-minor.

On combining Theorem 1.2 and (1.4) with the excluded-minor characterization of binary matroids, one easily gets that

(1.5) The 2-sum of $M_{1}$ and $M_{2}$ is binary if and only if both $M_{1}$ and $M_{2}$ are binary.

If $\{x, y\}$ is a circuit of the matroid $M$, we say that $x$ and $y$ are in parallel in $M$. If, instead, $\{x, y\}$ is a cocircuit, then $x$ and $y$ are in series. A parallel class of $M$ is a maximal subset $A$ of $E(M)$ such that if $a$ and $b$ are distinct elements of $A$, then $a$ and $b$ are in parallel. Series classes are defined analogously. A series or parallel class is nontrivial if it contains more than one element. The matroid $M^{\prime}$ is a series extension of $M$ if $M=M^{\prime} / T$ and every element of $T$ is in series with some element of $M$. Parallel extensions are defined analogously.

The following result of Bixby [2, Theorem 1] will be used repeatedly in the proof of the main theorem of this paper. 
(1.6) THEOREM. Let $e$ be an element of the 3-connected matroid $M$. Then either $M \backslash e$ is a series extension of a 3-connected matroid or $M / e$ is a parallel extension of a 3-connected matroid.

Suppose that $r \geq 3$. The wheel $\mathcal{W}_{r}$ of order $r$ is a graph having $r+1$ vertices, $r$ of which lie on a cycle (the rim); the remaining vertex is joined by a single edge (a spoke) to each of the other vertices. The whirl $\mathcal{W}^{r}$ of order $r$ is a matroid on $E\left(\mathcal{W}_{r}\right)$ having as its circuits all cycles of $\mathcal{W}_{r}$ other than the rim, as well as all sets of edges formed by adding a single spoke to the set of edges of the rim. The terms "rim" and "spoke" will be used in the obvious way in both $M\left(\mathcal{W}_{r}\right)$ and $\mathcal{W}^{r}$. It will also be convenient here to view the matroid $U_{2,4}$ as the whirl $\mathcal{W}^{2}$. Indeed, if one uses the construction of $\mathcal{W}^{r}$ from $M\left(\mathcal{W}_{r}\right)$ in the case when $r=2$, it is easy to see that the resulting matroid is isomorphic to $U_{2,4}$.

The following result of Tutte $[\mathbf{1 1}, 8.3]$ indicates the fundamental role that whirls and the cycle matroids of wheels play in the class of 3-connected matroids.

(1.7) THEOREM. Let $M$ be a 3-connected matroid such that for all elements e neither $M \backslash e$ nor $M / e$ is 3-connected. Then $M$ has rank at least three and is a whirl or the cycle matroid of a wheel.

An important tool in the proof of Theorem 1.7 and one that will also be needed in this paper is the following.

(1.8) LEMMA $[\mathbf{1 1}, 7.3]$. Let $M$ be a 3-connected matroid having at least four elements. Suppose that $\{a, b, c\}$ is a triad of $M$ such that neither $M / a$ nor $M / b$ is 3-connected. Then $M$ has a triangle containing $a$ and just one of $b$ and $c$.

The next theorem, the main result of this paper, will be proved in $\S 4$.

(1.9) THEOREM. Let $M$ be a 3-connected nonbinary matroid and suppose that $\{x, y, z\} \subseteq E(M)$. Then either $M$ has a $U_{2,4}$-minor using $\{x, y, z\}$ or $M$ has a $\mathcal{W}^{3}$-minor in which $\{x, y, z\}$ is the rim or the set of spokes.

A 3-connected matroid $M$ is internally 4-connected if $M$ has no exact 3-separation $\left(X_{1}, X_{2}\right)$ with $\left|X_{1}\right|,\left|X_{2}\right| \geq 4$. In $[\mathbf{1 0}]$, Seymour characterized precisely when three elements in a 3 -connected, internally 4-connected binary matroid are in a circuit. He also noted that the corresponding nonbinary problem is still open. As an immediate consequence of Theorem 1.9, we have the following result for the nonbinary problem.

(1.10) COROLlaRY. Let $M$ be a 3-connected nonbinary matroid and suppose that $\{x, y, z\} \subseteq E(M)$. Then $M$ has a circuit containing $\{x, y, z\}$ unless $M$ has a $W^{3}$-minor in which $\{x, y, z\}$ is the set of spokes.

$\S \S 2$ and 3 of this paper contain several results which are used in the proof of Theorem 1.9. Most of these results are also of interest in their own right. In particular, $\S 3$ proves the analogue of the main theorem for arbitrary 3-connected matroids.

2. A whirls theorem. In this section we shall use P. D. Seymour's theory of splitters [7] to prove that a nonbinary 3-connected matroid can be obtained from a whirl by a sequence of nontrivial lifts and extensions. The author's original proof of this result did not use splitters and he is indebted to Dr. Seymour for informing 
him that the results of this section were already known and could be easily derived using splitters.

Let $\mathcal{F}$ be a class of matroids closed under minors and under isomorphism. A member $N$ of $\mathcal{F}$ is a splitter for $\mathcal{F}$ if every 3-connected member of $\mathcal{F}$ having an $N$-minor is isomorphic to $N$.

The next result is Seymour's characterization of splitters $[\mathbf{7},(7.3)]$.

(2.1) THEOREM. Let $₹$ be a class of matroids closed under minors and under isomorphism. Suppose that $N$ is a nonempty connected member of $\mathcal{F}$ for which the following conditions hold:

(i) both $N$ and $N^{*}$ are simple;

(ii) $₹$ contains no nontrivial extensions of $N$ and no nontrivial lifts of $N$;

(iii) if $N \simeq M\left(\mathcal{W}_{k}\right)$ for some $k \geq 3$, then $M\left(\mathcal{W}_{k+1}\right) \notin \mathcal{F}$; and

(iv) if $N \simeq \mathcal{W}^{k}$ for some $k \geq 2$, then $\mathcal{W}^{k+1} \notin \mathcal{F}$.

Then $N$ is a splitter for $\mathcal{F}$.

An easy consequence of this is the following.

(2.2) THEOREM. Let $M$ and $N$ be 3-connected matroids such that $N$ is a minor of $M,|E(N)| \geq 4$, and if $N \simeq M\left(\mathcal{W}_{k}\right), M$ has no $M\left(\mathcal{W}_{k+1}\right)$-minor, while if $N \simeq \mathcal{W}^{k}, M$ has no $\mathcal{W}^{k+1}$-minor. Then there is a sequence $N_{0}, N_{1}, N_{2}, \ldots, N_{n}$ of 3 connected matroids such that $N_{0} \simeq N, N_{n}=M$, and, for all $i$ in $\{0,1,2, \ldots, n-1\}$, $N_{i}$ is a single-element deletion or contraction of $N_{i+1}$.

PROOF. Let $₹$ be the class of matroids $M_{1}$ that are isomorphic to some minor of $M$. Then $\mathcal{F}$ is closed under minors and under isomorphism. Let $M^{\prime}$ be a 3 connected member of $₹$ which is maximal with the property that there is a sequence $M_{0}^{\prime}, M_{1}^{\prime}, \ldots, M_{m}^{\prime}$ of 3 -connected matroids such that $M_{0}^{\prime} \simeq N, M_{m}^{\prime}=M^{\prime}$, and, for all $i$ in $\{0,1,2, \ldots, m-1\}, M_{i}^{\prime}$ is a single-element deletion or contraction of $M_{i+1}^{\prime}$. Then, as $|E(N)| \geq 4$, Theorem 3.1 implies that $M^{\prime}$ is a splitter for $\mathcal{F}$. Hence $M^{\prime} \simeq M$.

The following result for whirls, an immediate consequence of the last theorem, will be used in the proof of Theorem 1.9.

(2.3) COROLlaRY. Let $M$ be a nonbinary 3-connected matroid. Then $M$ can be obtained from a whirl by a sequence of nontrivial lifts and nontrivial extensions.

3. Some structural results. This section contains a number of results which will be needed in the proof of the main theorem. The first of these is a natural extension of Theorem 2.5 of [6] for nonbinary matroids. Euclidean representations for the 6-element matroids $P_{6}$ and $Q_{6}$ are shown in Figure 1.

(3.1) THEOREM. Let $M$ be a 3-connected nonbinary matroid having rank and corank at least three. Then $M$ has a minor isomorphic to one of $U_{3,6}, P_{6}, Q_{6}$, or $W^{3}$.

The proof of this theorem will use the following two lemmas.

(3.2) LEMMA. Let $N_{1}$ be a 3-connected matroid having an element e such that $N_{1} / e \simeq U_{2, k}$ for some $k \geq 5$. Then $N_{1}$ has a minor isomorphic to one of $U_{3,6}, P_{6}$, or $Q_{6}$.

ProOF. We argue by induction on $k$. If $k=5$, then $N_{1}$ is a nontrivial lift of $U_{2,5}$, and it is not difficult to check that $N_{1}$ is isomorphic to one of $U_{3,6}, P_{6}$, or $Q_{6}$. Thus 

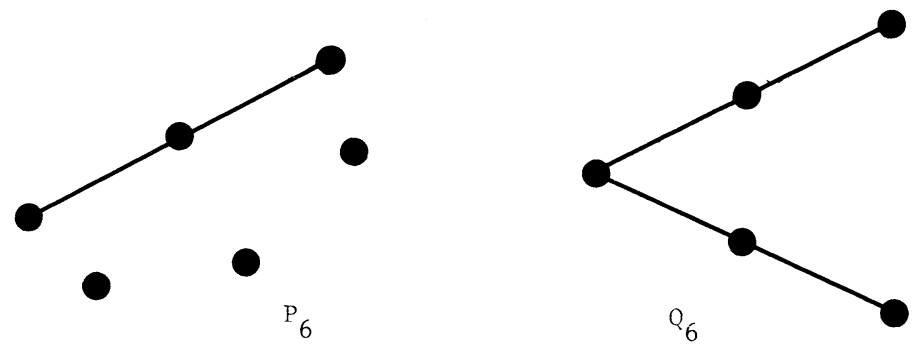

FIGURE 1

the proposition is true for $k=5$. Now assume it true for $k=n$ where $n \geq 5$ and let $k=n+1$. Suppose that $f$ is an element of $E\left(N_{1} / e\right)$. Then $N_{1} \backslash f / e \simeq U_{2, n}$ and the induction assumption implies that, provided $N_{1} \backslash f$ is 3-connected, $N_{1} \backslash f$ and hence $N_{1}$ has a minor isomorphic to one of $U_{3,6}, P_{6}$, or $Q_{6}$. Thus we may assume that, for all elements $f$ of $E\left(N_{1}\right)-\{e\}, N_{1} \backslash f$ is not 3-connected. But, both $N_{1}$ and $N_{1} \backslash f / e$ are 3-connected and so, by [5, Lemma 2.6], $N_{1}$ has a triad containing $\{e, f\}$. Therefore $N_{1} \backslash e$ is not 3-connected and so $N_{1}$ is minimally 3-connected. Since rk $N_{1}=3$, it follows by $\left[\mathbf{5}\right.$, Theorem 4.7] that $\left|E\left(N_{1}\right)\right| \leq 6$, a contradiction. This completes the proof of the lemma.

(3.3) LEMMA. Let $M$ be a 3-connected matroid having rank at least three and suppose that $M$ has a $U_{2,5}$-minor. Then $M$ has a 3-connected minor $N_{1}$ which has a single-element contraction that is isomorphic to $U_{2, k}$ for some $k \geq 5$.

PROOF. Let $k$ be the greatest integer $m$ for which $M$ has a $U_{2, m}$-minor. Then $k \geq 5$. Now let $N$ be a $U_{2, k}$-minor of $M$ and apply Theorem 2.2. The choice of $N$ guarantees that no 3-connected extension of it is a minor of $M$. It follows that $M$ has as a minor a 3-connected lift $N_{1}$ of $N$.

Proof of TheOREM 3.1. Since $M$ is nonbinary, it has a $U_{2,4}$-minor $N$. Now clearly $U_{2,5}$ is the only nontrivial extension of $U_{2,4}$ and, by duality, $U_{3,5}$ is the only nontrivial lift. Thus, on applying Theorem 2.2 and recalling that $U_{2,4} \simeq \mathcal{W}^{2}$, we obtain that $M$ has a minor isomorphic to one of $W^{3}, U_{2,5}$, or $U_{3,5}$. In the first case, the theorem is immediate, while in the second and third cases, it follows on applying the combination of Lemmas 3.2 and 3.3 to $M$ and $M^{*}$ respectively.

Theorem 3.1 can be extended by using the following result.

(3.4) LeMmA. Let $M$ be a 3-connected matroid and $\{x, y\}$ be a subset of $E(M)$. Suppose that $M$ has a minor isomorphic to a member of $\left\{U_{3,6}, P_{6}, Q_{6}, \mathcal{W}^{3}\right\}$. Then $M$ has such a minor using $\{x, y\}$.

PROOF. We use the main result of Seymour's paper [9]. As each of $U_{3,6}, P_{6}, Q_{6}$, and $\mathcal{W}^{3}$ is self-dual, we need only check that if $M$ is a 3-connected matroid such that $M \backslash e$ is isomorphic to one of the four specified matroids and $f \in E(M \backslash e)$, then $M$ has a deletion using $\{e, f\}$ that is isomorphic to one of the four specified matroids. We omit the straightforward checking of cases that is needed to complete the proof.

On combining this lemma with Theorem 3.1, we immediately obtain the following. 
(3.5) COROLlARY. Let $M$ be a 3-connected nonbinary matroid having rank and corank at least three and suppose that $\{x, y\} \subseteq E(M)$. Then $M$ has a minor isomorphic to one of $U_{3,6}, P_{6}, Q_{6}$, or $\mathcal{W}^{3}$ that uses $\{x, y\}$.

The next result corresponds to the main theorem in the case that one does not restrict to nonbinary matroids.

(3.6) THEOREM. Let $M$ be a 3-connected matroid having rank and corank at least three and suppose that $\{x, y, z\} \subseteq E(M)$. Then $M$ has a minor isomorphic to one of $U_{3,6}, P_{6}, Q_{6}, \mathcal{W}^{3}$, or $M\left(K_{4}\right)$ that uses $\{x, y, z\}$.

Proof. As $M$ is 3-connected having rank and corank at least three, $|E(M)| \geq 6$ and, by Theorem 2.5 of [6], $M$ has a minor isomorphic to one of the five specified matroids. The result therefore follows immediately if $|E(M)|=6$. We shall argue by induction on $|E(M)|$ so suppose that $|E(M)|>6$ and that the result is true for all matroids with fewer elements than $M$. Let $\operatorname{rk} M=3$. If $M$ has no element $e$ such that $M \backslash e$ is 3-connected, then, by [5, Theorem 4.7], $|E(M)| \leq 6$; a contradiction. Hence, $M$ does have such an element $e$. If $e \notin\{x, y, z\}$, then the result follows by the induction assumption. Thus we may suppose, without loss of generality, that $e=x$. By [6, Theorem 4.2], $M \backslash x$ has a restriction isomorphic to one of $U_{3,6}$, $P_{6}, Q_{6}, \mathcal{W}^{3}$, or $M\left(K_{4}\right)$ that uses $\{y, z\}$. It is now a straightforward matter to check that in all five of these cases the required result holds. We note that the case checking required here is very similar to that required in the proof of Lemma 3.4. We conclude that the theorem holds if $M$ has rank 3 and, by duality, it also holds if $M$ has corank 3 . We shall now assume that both the rank and corank of $M$ exceed 3 .

By Theorem 1.6, for all elements $e$ of $M$, either $M \backslash e$ is a series extension of a 3-connected matroid or $M / e$ is a parallel extension of a 3-connected matroid. Choose $e$ in $E(M)-\{x, y, z\}$ and suppose that $M \backslash e$ is a series extension of the 3 -connected matroid $N$ and that $x, y$, and $z$ are in different series classes of $N$. Then we may assume that $\{x, y, z\} \subseteq E(N)$. Now either

(i) $N$ has rank at least 3 ; or

(ii) $N$ has rank 2 .

In both cases, since cork $M>3$, cork $N \geq 3$. The result therefore follows in the first case by applying the induction assumption to $N$. In the second case, $N \simeq U_{2, k}$ for some $k$ and, since $\operatorname{cork} N \geq 3, k \geq 5$. Now as $\operatorname{rk} M \geq 4$ and $\operatorname{rk} N=2, M \backslash e$ has at least one nontrivial series class. Choose $x_{1}$ and $x_{2}$ in this class taking $x_{1}$ equal to $x, y$, or $z$ if one of these elements is in the class. Contract all the elements from this class except $x_{1}$ and $x_{2}$ and then contract all but one element from each of the other nontrivial series classes of $M \backslash e$ ensuring that $x, y$, and $z$ are kept. The resulting contraction of $M$ has rank 3 and has $\left\{e, x_{1}, x_{2}\right\}$ as a cocircuit. Now delete all but three elements, $a, b$, and $c$, from the line which is complementary to $\left\{e, x_{1}, x_{2}\right\}$ again ensuring that $x, y$, and $z$ are kept.

In the resulting matroid $M^{\prime}$, the closure of $\left\{x_{1}, x_{2}\right\}$ does not contain $e$ otherwise $e$ is in the closure in $M$ of the series class $S_{1}$ of $M \backslash e$ containing $\left\{x_{1}, x_{2}\right\}$. In that case, $\operatorname{rk}\left(S_{1} \cup e\right)=\operatorname{rk} S_{1}$ and if $S_{2}=E(M \backslash e)-S_{1}$, then $\operatorname{rk} M=\operatorname{rk}(M \backslash e)=\operatorname{rk} S_{2}+\left|S_{1}\right|-1$ and $\operatorname{rk} S_{1}=\left|S_{1}\right|$. Thus $\operatorname{rk} S_{1}+\operatorname{rk} S_{2}=\operatorname{rk} M+1$, so $\operatorname{rk}\left(S_{1} \cup e\right)+\operatorname{rk} S_{2}=\operatorname{rk} M+1$, a contradiction to the fact that $M$ is 3-connected. We conclude that, in $M^{\prime},\left\{e, x_{1}, x_{2}\right\}$ 


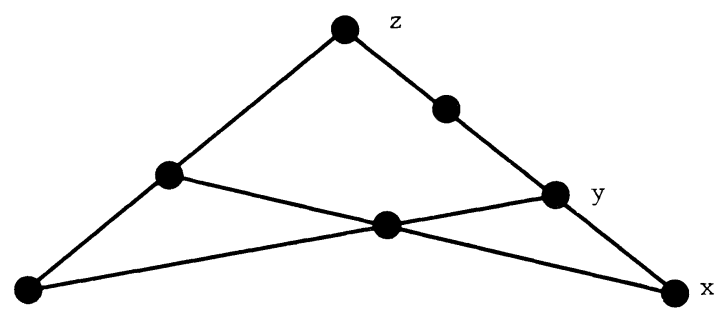

FIGURE 2

is a basis. It follows without difficulty that $M^{\prime}$ is 3 -connected, hence the required result holds in case (ii).

We may now assume that, for all elements $e$ of $E(M)-\{x, y, z\}$, either at least two of $x, y$, and $z$ are in series in $M \backslash e$, or at least two of $x, y$, and $z$ are in parallel in $M / e$. If $x$ and $y$ are in series in $M \backslash f_{1}$ and $x$ and $z$ are in parallel in $M / f_{2}$ where $f_{2} \neq f_{1}$, then $\left\{x, y, f_{1}\right\}$ is a cocircuit of $M$ and $\left\{x, z, f_{2}\right\}$ is a circuit of $M$. But $\left|\left\{x, y, f_{1}\right\} \cap\left\{x, z, f_{2}\right\}\right|=1$; a contradiction.

Suppose next that $x$ and $y$ are in series in both $M \backslash f_{1}$ and $M \backslash f_{2}$. Then $\left\{x, y, f_{1}\right\}$ and $\left\{x, y, f_{2}\right\}$ are cocircuits of $M$ and hence, by exchange, $\left\{x, f_{1}, f_{2}\right\}$ and $\left\{y, f_{1}, f_{2}\right\}$ are cocircuits of $M$. It follows then that, for $f_{3}$ in $E(M)-\left\{x, y, z, f_{1}, f_{2}\right\}$, no pair of $x, y$, and $z$ can be in a parallel class in $M / f_{3}$, otherwise we will again get a circuit and a cocircuit of $M$ having exactly one common element.

As $|E(M)-\{x, y, z\}| \geq 5$, it follows that either, for all $e$ in $E(M)-\{x, y, z\}$, at least two of $x, y$, and $z$ are in series in $M \backslash e$, or, for all such $e$, at least two of $x, y$, and $z$ are in parallel in $M / e$. In the first case, $\{x, y, z\}$ is spanning in $M^{*}$; in the second case, it is spanning in $M$. Thus we obtain the contradiction that $M$ has rank or corank at most 3 , and this completes the proof of the theorem.

The next result comes from applying the last theorem to binary matroids.

(3.7) COROLlaRY. Let $M$ be a 3-connected binary matroid having rank and corank at least three and suppose that $\{x, y, z\} \subseteq E(M)$. Then $M$ has a minor isomorphic to $M\left(K_{4}\right)$ that uses $\{x, y, z\}$.

In view of this result it seems tempting to conjecture the following extension of Corollary 3.5: If $M$ is a 3-connected nonbinary matroid having rank and corank at least three and $\{x, y, z\} \subseteq E(M)$, then $M$ has a minor isomorphic to one of $U_{3,6}$, $P_{6}, Q_{6}$, or $\mathcal{W}^{3}$ using $\{x, y, z\}$. However, the matroid shown in Figure 2 satisfies the hypotheses of this conjecture but not its conclusion. Theorem 1.9 arises fairly naturally when one attempts to modify this conjecture in light of the above example.

The next result characterizes those connected nonbinary matorids having an element which is used by every $U_{2,4}$-minor.

(3.8) THEOREM. Let $M$ be a nonbinary connected matroid such that for some element e of $M$ both $M \backslash e$ and $M / e$ are binary. Then $M$ is obtained from a 4-point line having ground set $\left\{e, e_{1}, e_{2}, e_{3}\right\}$ by a sequence of at most three 2 -sums where the basepoints of these 2-sums are $e_{1}, e_{2}$, and $e_{3}$, the other part of each 2-sum is connected and binary, and each of $e_{1}, e_{2}$, and $e_{3}$ is the basepoint of at most one of these 2-sums.

PROOF. Evidently $M$ has rank and corank at least two. We shall suppose first that $M$ is 3 -connected. If $M$ has rank two, then $M \simeq U_{2, k}$ for some $k \geq 4$. If 
$k>4$, then $M$ does not have the required property. Thus if $M$ has rank 2 , then the required result holds. By duality, if $M$ has corank two, then $M^{*} \simeq U_{2,4}$ and so $M \simeq U_{2,4}$.

We may now assume that if $M$ is 3 -connected, then $\operatorname{rk} M$, cork $M \geq 3$. In that case, by Corollary 3.5, $M$ has a minor $N$ isomorphic to one of $U_{3,6}, P_{6}, Q_{6}$, or $W^{3}$ that uses $e$. It is straightforward to check that, in each case, one of $N \backslash e$ and $N / e$ is nonbinary. Hence we obtain the contradiction that one of $M \backslash e$ and $M / e$ is nonbinary.

We may now assume that $M$ is not 3-connected. Then

$$
M=P\left(\left(M_{1}, p_{1}\right),\left(M_{2}, p_{2}\right)\right) \backslash p
$$

for some matroids $M_{1}$ and $M_{2}$. If $M_{i}$ is nonbinary for $i=1$ and 2 , then as $M_{i}$ is connected, by $\left[\mathbf{1}\right.$, Theorem 3.7], it has a $U_{2,4}$-minor using $p_{i}$. Thus $M$ has a minor isomorphic to the 2-sum of two copies of $U_{2,4}$ and so one of $M \backslash e$ and $M / e$ is nonbinary; a contradiction. It follows that we may assume that $M_{1}$ is nonbinary and $M_{2}$ is binary and that $\left|E\left(M_{2}\right)\right|$ is maximum subject to these conditions. Clearly $e \in E\left(M_{1}\right)$. If $M_{1}$ is 3 -connected, then $M_{1} \simeq U_{2,4}$. If $M_{1}$ is not 3 -connected, it is the 2-sum of a nonbinary and a binary matroid. Again choose the binary part of $M_{1}$ to have cardinality as large as possible. If the nonbinary part of $M_{1}$ is not 3 -connected, then decompose it. We may repeat such decompositions, in each case choosing the binary part to have maximum cardinality, until we obtain $U_{2,4}$ as one of the parts of the 2 -sum. The other part of this 2 -sum must be a connected binary matroid. One of the three elements of $U_{2,4}$ different from $e$ is the basepoint of this 2-sum. In building back up to $M$, it follows, by the fact the cardinality of the binary part of each 2 -sum was chosen to be maximum, that $M$ can indeed be obtained from $U_{2,4}$ as described.

(3.9) COROLLARY. Let $M$ be a 3-connected nonbinary matroid having an element $e$ such that both $M \backslash e$ and $M / e$ are binary. Then $M \simeq U_{2,4}$.

4. The main theorem. In this section we shall prove Theorem 1.9 , the main result of this paper. This theorem extends the following result of Seymour [8, (3.1)].

(4.1) THEOREM. Let $M$ be a nonbinary 3-connected matroid and suppose that $\{x, y\} \subseteq E(M)$. Then $M$ has a $U_{2,4}$-minor using $\{x, y\}$.

The proof of Theorem 1.9 is a long induction argument and will be presented as a sequence of lemmas. The basic idea of the proof is to find, in a minimal counterexample $M$, elements $f$ that are not in $\{x, y, z\}$, such that both $M \backslash f$ and $M / f$ are nonbinary. Given such an element, we apply Theorem 1.6 to get that either $M \backslash f$ is a nonbinary series extension of a 3-connected matroid or $M / f$ is a nonbinary parallel extension of a 3-connected matroid. But then the induction assumption can be applied to one of $M \backslash f$ and $M^{*} \backslash f$ unless $x, y$, and $z$ do not all lie in different series classes. In the exceptional case, if one has sufficiently many such elements $f$, then one can deduce enough about the structure of $M$ to get the theorem. In order to find elements $f$ with the required property, we shall rely on the following lemma.

(4.2) LEMMA. Suppose that $M$ is the 2-sum of $M_{1}$ and $M_{2}$ where $M_{2}$ is nonbinary. If $f$ is an element of $M_{1}$ that is neither in series nor in parallel with $p_{1}$, then both $M \backslash f$ and $M / f$ are nonbinary. 


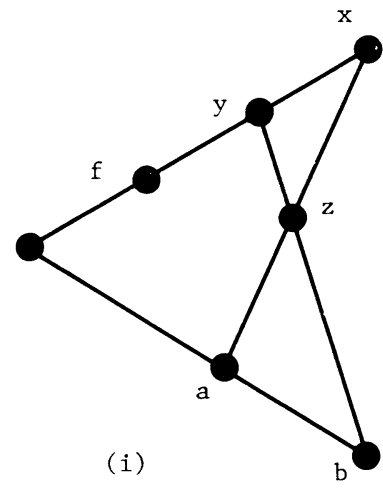

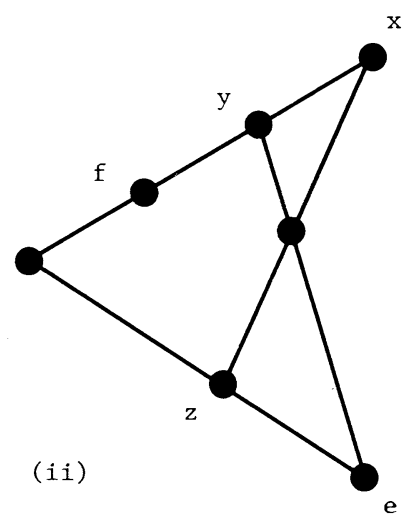

FIGURE 3

PROOF. The result is immediate if $f$ and $p_{1}$ are in different components of $M_{1}$. Thus assume that $f$ and $p_{1}$ are in the same component of $M_{1}$. As $f$ and $p_{1}$ are not in series, $M_{1}$ has a circuit containing $p_{1}$ and avoiding $f$. Thus $M \backslash f$ has an $M_{2}$-minor and so is nonbinary. On applying the same argument to $M^{*}$, we get that $M^{*} \backslash f$ and hence $M / f$ is nonbinary.

The next lemma proves the theorem in the case that two of $x, y$, and $z$ lie on a line in $M$ having at least four points.

(4.3) LEMMA. If $M$ satisfies the hypotheses of Theorem 1.9 and, in addition, has a restriction isomorphic to $U_{2,4}$ that uses two of $x, y$, and $z$, then $M$ satisfies the conclusion of Theorem 1.9 .

ProOF. We shall suppose that $M$ has a $U_{2,4}$-restriction using $\{x, y\}$. If $M$ has rank 2 , then the lemma is immediate. Since $M$ has $U_{2,4}$ as a restriction, $M$ cannot have corank 2 unless $M \simeq U_{2,4}$. It follows that we may assume that both the rank and corank of $M$ exceed two. Then as $M$ is 3-connected, by Theorem 3.6, $M$ has a minor $N$ isomorphic to one of $M\left(K_{4}\right), U_{3,6}, W^{3}, P_{6}$ or $Q_{6}$ that uses $\{x, y, z\}$. In the last four cases, it is routine to check that the lemma holds. We note, for future reference, that in these cases the existence of a $U_{2,4}$-restriction of $M$ using two of $x, y$, and $z$ is not needed to obtain the conclusion. In the remaining case, $N \simeq M\left(K_{4}\right)$.

Let $L$ be the line of $M$ containing $\{x, y\}$. Then, in forming $N$ from $M$, no element of $L$ can be contracted. The lemma is immediate if $z \in L$, so we assume that this is not so. If $x$ and $y$ are on one of the 3-point lines of $N$, then choose an element $f$ of $L-E(N)$ and let $N^{\prime}$ be the extension of $N$ by $f$. Without loss of generality, we may assume that $x, y$, and $z$ are as shown in Figure 3(i) or (ii). In the first case, on labelling the elements $a$ and $b$ as shown, we get that $N^{\prime} \mid\{x, y, z, a, b, f\} \simeq \mathcal{W}^{3}$ and has $\{x, y, z\}$ as its set of spokes. In the second case, it is clear that $N^{\prime} / e$ has a $U_{2,4}$-restriction using $\{x, y, z\}$.

If $x$ and $y$ are not on one of the 3-point lines of $N$, then we extend $N$ by adding two elements of $L-E(N)$. This can be done in several different ways but it is routine to show that, in each case, $M$ has a $U_{2,4}$-minor using $\{x, y, z\}$.

PROOF OF THEOREM 1.9. Let $M$ be a nonbinary 3 -connected matroid which is a minor-minimal counterexample to the theorem. Then $M^{*}$ is also a minimal 


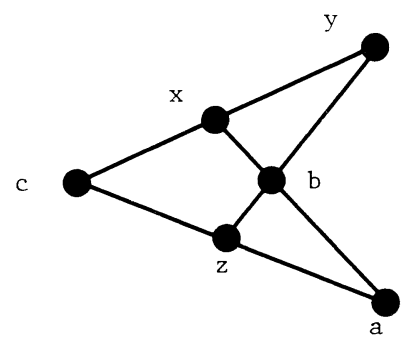

FIGURE 4

counterexample to the theorem. If $\operatorname{rk} M=2$, then $M \simeq U_{2, k}$ for some $k \geq 4$ and so the theorem holds for $M$; a contradiction. By duality, if cork $M=2$, then the theorem also holds for $M$. Thus $\operatorname{rk} M, \operatorname{cork} M \geq 3$. Suppose that $M$ has rank 3 . Then, by Theorem 3.6, $M$ has a restriction isomorphic to one of $U_{3,6}, \mathcal{W}^{3}, P_{6}, Q_{6}$, or $M\left(K_{4}\right)$ that uses $\{x, y, z\}$. It was noted in the preceding proof that, in the first four cases, the theorem holds. Thus $M$ has a restriction $N$ isomorphic to $M\left(K_{4}\right)$ that uses $\{x, y, z\}$. Since $M$ is nonbinary, it is not isomorphic to $M\left(K_{4}\right)$ or the Fano matroid. It therefore has an element $e$ such that the extension $N^{\prime}$ of $N$ by $e$ is nonbinary. Evidently $N^{\prime}$ is 3-connected. Therefore, as $N^{\prime} \backslash e$ is binary, Corollary 3.9 implies that $N^{\prime} / e$ is nonbinary. But $N^{\prime} / e$ has rank two and therefore has a $U_{2,4}$-restriction using $\{x, y, z\}$ unless $e$ is collinear with two of $x, y$, and $z$ in $N^{\prime}$. By Lemma 4.3, $N^{\prime}$ has no 4-point line using two of $x, y$, and $z$. It follows that, without loss of generality, we may assume that $x, y$, and $z$ are as shown in Figure 4 , and that $e, x$, and $z$ are collinear. Now $e, a$, and $y$ must also be collinear, otherwise $N^{\prime} / a$ has a $U_{2,4}$-restriction using $\{x, y, z\}$ and the theorem holds for $M$; a contradiction. Furthermore, $b, c$, and $e$ are not collinear, otherwise $N^{\prime}$ is isomorphic to the Fano matroid and hence is binary. Thus $N^{\prime} \mid\{x, y, z, b, c, e\} \simeq \mathcal{W}^{3}$ and has $\{x, y, z\}$ as its set of spokes.

We conclude that if $M$ has rank 3 and, by duality, if $M$ has corank 3, then the theorem holds for $M$; a contradiction. Thus both the rank and corank of $M$ exceed 3 .

As $M$ is nonbinary and 3-connected, by Corollary 2.3, $M$ is obtained from a whirl by a sequence of nontrivial lifts and nontrivial extensions. Now $M$ itself is not a whirl, otherwise, as is easily checked, it is not a counterexample to the theorem. Thus $M$ has an element $e$ such that either $M \backslash e$ or $M / e$ is obtained from a whirl in the manner described. We may assume that the first of these occurs, otherwise we replace $M$ by $M^{*}$ in the argument that follows. If $e \notin\{x, y, z\}$, then we get a contradiction by applying the induction assumption to $M \backslash e$. Thus, we can assume that $e=x$. Notice that this assumption distinguishes $x$ from $y$ and $z$.

The rest of the proof of Theorem 1.9 will consist of a sequence of eight lemmas. For some of these, both the lemma and its dual will be used in the proof of the theorem.

(4.4) LEMMA. Suppose that $a$ is an element of $E(M)-\{x, y, z\}$ for which $M \backslash a$ is a nonbinary series extension of a 3-connected matroid. Then a is the unique such element. Moreover, $y$ and $z$ are in the same series class of $M \backslash a$, this class does not contain $x$, and $\{a, y, z\}$ is a triad of $M$. 
ProOF. Let $M \backslash a$ be a series extension of the 3-connected matroid $N$. If $x, y$, and $z$ are in distinct series classes of $M \backslash a$, then we may assume that $\{x, y, z\} \subseteq E(N)$. Since the theorem holds for $N$, it follows that it also holds for $M$; a contradiction. If $x, y$, and $z$ are all in the same series class of $M \backslash a$, then every 3-element subset of $\{a, x, y, z\}$ is a triad of $M$. Thus $M \cdot\{a, x, y, z\} \simeq U_{2,4}$; a contradiction. This leaves the possibility that two of $x, y$, and $z$ are in the same series class of $M \backslash a$, while the third is in a different series class. As $M \backslash x$ is 3-connected, $x$ is not in a triad of $M$ and therefore $x$ is not in a 2-cocircuit of $M \backslash a$. Thus $\{y, z\}$ is a cocircuit of $M \backslash a$ and hence $\{a, y, z\}$ is a cocircuit of $M$. To establish the uniqueness of $a$, suppose that $M \backslash b$ is a nonbinary series extension of a 3 -connected matroid where $b \in E(M)-\{x, y, z, a\}$. Then, applying the above argument with $b$ replacing $a$, we get that $\{b, y, z\}$ is a cocircuit of $M$ and so $M^{*} \mid\{a, b, y, z\} \simeq U_{2,4}$. It now follows by applying Lemma 4.3 to $M^{*}$ that the conclusion of the theorem holds for $M^{*}$ and hence for $M$; a contradiction.

(4.5) LemMA. Suppose that $a \in E(M)-\{x, y, z\}$ and $M \backslash a$ is a nonbinary series extension of a 3-connected matroid. Then $M$ has no element $b$ different from $a, x, y$, and $z$ such that both $M \backslash b$ and $M / b$ are nonbinary.

Proof. Assume that $M$ has such an element $b$. By the preceding lemma, $M \backslash b$ is not a series extension of a 3 -connected matroid. Thus, by Theorem 1.6, $M / b$ is a parallel extension of a 3-connected matroid. By dualizing the argument given in the preceding lemma, it follows that we may suppose that two of $x, y$, and $z$ lie in the same parallel class of $M / b$ while the third lies in a different parallel class. Thus $\{b, g, h\}$ is a triangle of $M$ for some subset $\{g, h\}$ of $\{x, y, z\}$. As $\{a, y, z\}$ is a triad of $M$, it follows that $\{g, h\}=\{y, z\}$, otherwise we have a triangle and a triad meeting in just a single element. Thus $\{b, y, z\}$ is a triangle of $M$.

We show next that $M \backslash b$ is a series extension of a 3-connected matroid. If not, then by [2, Lemma 2], $M \backslash b$ has an exact 2-separation $(Y, Z)$ in which both $|Y|$ and $|Z|$ exceed two. Now $\{y, z\} \nsubseteq Y$, otherwise $(Y \cup b, Z)$ is an exact 2-separation of the 3-connected matroid $M$; a contradiction. Likewise, $\{y, z\} \nsubseteq Z$. Thus we may assume that $y \in Y$ and $z \in Z$. We may also suppose that $a \in Y$. Then, as $\{a, y, z\}$ is a triad of $M, z$ is a coloop of $M \mid Z$. Hence $\operatorname{rk}(Z-z)=\operatorname{rk} Z-1$. In addition, $\operatorname{rk}(Y \cup\{z, b\})=\operatorname{rk}(Y \cup z)$ and therefore

$$
\operatorname{rk}(Y \cup\{z, b\})+\operatorname{rk}(Z-z)=\operatorname{rk}(Y \cup z)+\operatorname{rk}(Z-z) \leq \operatorname{rk} Y+\operatorname{rk} Z=\operatorname{rk} M+1 .
$$

Since $M$ is 3 -connected, it follows that $|Z-z| \leq 1$, that is, $|Z| \leq 2$; a contradiction. We conclude that $M \backslash b$ is indeed a series extension of a 3 -connected matroid. But $M \backslash b$ is also nonbinary and so we have a contradiction to Lemma 4.4.

(4.6) LEMMA. $M$ does not have three distinct elements $a, b$, and $c$ that are not in $\{x, y, z\}$ such that both $M \backslash e$ and $M / e$ are nonbinary for all $e$ in $\{a, b, c\}$.

Proof. Assume that $M$ does have three such elements, $a, b$, and $c$. Then, by Lemma 4.5, none of $M \backslash a, M \backslash b$, or $M \backslash c$ is a series extension of a 3-connected matroid. Therefore, by Theorem 1.6, each of $M / a, M / b$ and $M / c$ is a parallel extension of a 3-connected matroid. We obtain the contradiction that the theorem holds for $M$ unless in each of $M / a, M / b$, and $M / c$, two of $x, y$, and $z$ lie in the same parallel class while the third lies in a different parallel class. If $\{j, k\} \subseteq\{x, y, z\}$ and $\{j, k\}$ lies in a parallel class in two of $M / a, M / b$, and $M / c$, then, by Lemma 
4.3, the theorem holds for $M$. Thus, we may assume that $x$ and $y$ are parallel in $M / a$, that $y$ and $z$ are parallel in $M / b$, and that $x$ and $z$ are parallel in $M / c$. Then $\{a, x, y\},\{b, y, z\}$, and $\{c, x, z\}$ are triangles of $M$. The theorem follows unless $M \mid\{a, b, c, x, y, z\} \simeq M\left(K_{4}\right)$ and $\{a, b, c\}$ is also a triangle of $M$. In that case, as none of $M \backslash a, M \backslash b$, or $M \backslash c$ is 3-connected, the dual of Lemma 1.8 implies that $M$ has a triad $T_{a}$ containing $a$ and just one of $b$ and $c$. If $c \in T_{a}$, then, from the intersection of $T_{a}$ with the triangles $\{a, x, y\}$ and $\{c, x, z\}$, we get the contradiction that $x \in T_{a}$. Thus $b \in T_{a}$ and, from considering intersections with the triangles $\{a, x, y\}$ and $\{b, y, z\}$, we get that $T_{a}=\{a, b, y\}$. By a similar argument, we have that $M$ has a triad $T_{c}$ containing $c$ and just one of $a$ and $b$, and hence that $T_{c}=\{c, b, z\}$. Now

$$
\operatorname{rk}\left(T_{a} \cup T_{c}\right)+\operatorname{rk}\left(E(M)-\left(T_{a} \cup T_{c}\right)\right)-\operatorname{rk} M \leq 3+\operatorname{rk} M-2-\operatorname{rk} M=1 .
$$

But $M$ is 3-connected, hence $\left|E(M)-\left(T_{a} \cup T_{c}\right)\right| \leq 1$. It follows that $|E(M)|=6$ and $M \simeq M\left(K_{4}\right)$; a contradiction to the fact that $M$ is nonbinary.

(4.7) LEMMA. Suppose that $M$ has an element e different from $x, y$, and $z$ such that $M \backslash e$ is nonbinary and is not a series extension of a 3-connected matroid. Then $M$ has two distinct elements $f_{1}$ and $f_{2}$ that are not in $\{x, y, z\}$ such that all of $M \backslash f_{1}, M / f_{1}, M \backslash f_{2}$, and $M / f_{2}$ are nonbinary.

The next two rather straightforward lemmas will be used in the proof of Lemma 4.7 .

(4.8) LEMMA. Let $N$ be a 3-connected matroid. Suppose that $e \in E(N)$ and $N \backslash e$ is the 2-sum of $N_{1}$ and $N_{2}$ where $N_{2}$ is nonbinary. If $N_{1}$ has an element $q_{1}$ that is parallel to the basepoint $p_{1}$, then $N / e$ is nonbinary.

Proof. Let $E\left(N_{1}\right)-p_{1}=E_{1}$ and $E\left(N_{2}\right)-p_{2}=E_{2}$. By (1.3), $N_{2}$ is connected. Hence the closure of $E_{2}$ in $N$ contains $q_{1}$. But $N$ is 3-connected, so $e$ is not in the closure of $E_{2} \cup\left\{q_{1}\right\}$ in $N$. Therefore, in $N \backslash\left(E_{1}-\left\{q_{1}\right\}\right)$, the element $e$ is a coloop. Thus

$$
N / e \backslash\left(E_{1}-\left\{q_{1}\right\}\right) \simeq N \backslash e \backslash\left(E_{1}-\left\{q_{1}\right\}\right) \simeq N_{2} .
$$

Hence $N / e$ is nonbinary and the lemma is proved.

(4.9) LEMMA. Let $e$ be an element of $M$ different from $x, y$, and $z$ such that $M \backslash e$ is the 2-sum of two nonbinary matroids $M_{1}$ and $M_{2}$. Then $E(M)-\{x, y, z, e\}$ contains three distinct elements $a, b$, and $c$ such that both $M \backslash f$ and $M / f$ are nonbinary for all $f$ in $\{a, b, c\}$.

Proof. For $i$ in $\{1,2\}$, let $A_{i}$ be the subset of $E\left(M_{i}\right)-p_{i}$ consisting of those elements that are neither in series nor in parallel with $p_{i}$ in $M_{i}$. Then, since $M_{i}$ is nonbinary, $\left|A_{i}\right| \geq 3$. Thus $\left|\left(A_{1} \cup A_{2}\right)-\{x, y, z\}\right| \geq 3$. The lemma follows immediately since if $f \in A_{1} \cup A_{2}$, then, by Lemma 4.2 , both $M \backslash f$ and $M / f$ are nonbinary.

Proof of Lemma 4.7. As $M \backslash e$ is not a series extension of a 3-connected matroid,

$$
M \backslash e=P\left(\left(M_{1}, p_{1}\right),\left(M_{2}, p_{2}\right)\right) \backslash p
$$

where $M_{1}$ has at least four elements and is not a single circuit $[\mathbf{7},(5.1)(\mathrm{ii})]$ and $M_{2}$ is nonbinary. Moreover, it follows on combining Lemmas 4.6 and 4.9 that $M_{1}$ 
is binary. We shall prove Lemma 4.7 first in the case when $p_{1}$ is parallel with another element $q_{1}$ of $M_{1}$. As $M \backslash e$ has no 2-circuits, $\left\{p_{1}, q_{1}\right\}$ is a parallel class of $M_{1}$. Now $M \backslash q_{1}$ is nonbinary since it has $M_{2}$ as a minor. Moreover, $M \backslash e / q_{1}$ has rank at least 3 and is not connected, so $M / q_{1}$ cannot be a parallel extension of a 3-connected matroid. Thus, by Theorem 1.6, $M \backslash q_{1}$ is a series extension of a 3-connected matroid. As $M \backslash q_{1}$ is nonbinary, it follows by Lemma 4.4 that either

(I) $q_{1} \notin\{x, y, z\}$ and $\left\{q_{1}, y, z\right\}$ is a triad of $M$, or

(II) $q_{1} \in\{x, y, z\}$.

In case I, $\left\{q_{1}, y, z\right\}$ is also a triad of $M \backslash e$, otherwise $M^{*} \mid\left\{e, q_{1}, y, z\right\} \simeq U_{2,4}$ and we obtain a contradiction by applying Lemma 4.3 to $M^{*}$. As $\left\{p_{1}, q_{1}\right\}$ is a circuit of $M_{1},\left\{q_{1}, y, z\right\}$ is not a cocircuit of $M_{1}$. Thus $\left\{q_{1}, p_{1}\right\},\left\{q_{1}, y, p_{1}\right\}$, or $\left\{q_{1}, z, p_{1}\right\}$ is a cocircuit of $M_{1}$. But, as $M_{1}$ is connected and $\left|E\left(M_{1}\right)\right| \geq 4$, it cannot have $\left\{p_{1}, q_{1}\right\}$ as both a circuit and a cocircuit. Hence, without loss of generality, we may assume that $\left\{q_{1}, y, p_{1}\right\}$ is a cocircuit of $M_{1}$. Then $\left\{p_{2}, z\right\}$ is a cocircuit of $M_{2}$ and so $z \in E\left(M_{2}\right)$. If $E\left(M_{1}\right)=\left\{x, y, p_{1}, q_{1}\right\}$, then $x$ is in a 2-cocircuit of $M_{1}$ avoiding $p_{1}$ and we get the contradiction that $x$ is in a triad of $M$. Therefore, $E\left(M_{1}\right)-\left\{x, y, p_{1}, q_{1}\right\}$ is nonempty and, by Lemma 4.2 , if $f$ is in this set, then both $M \backslash f$ and $M / f$ are nonbinary. But $M \backslash q_{1}$ is a nonbinary series extension of a 3 -connected matroid. Hence, by Lemma 4.5, we obtain a contradiction. Thus case I cannot occur.

Consider case II. If we can show that $E\left(M_{1}\right)-\left\{x, y, z, p_{1}\right\}$ contains at least two elements, then, by Lemma 4.2 , we may take $f_{1}$ and $f_{2}$ to be two elements of this set. We note that we could take $f_{2}=e$ for $M \backslash e$ is certainly nonbinary and, by Lemma $4.8, M / e$ is also nonbinary. Thus to prove this lemma, we need only find a single element in $E\left(M_{1}\right)-\left\{x, y, z, p_{1}\right\}$. However, for use in the proof of Lemma 4.11, we shall determine when we may need to take one of $f_{1}$ and $f_{2}$ equal to $e$. If $E\left(M_{1}\right)=\left\{x, y, z, p_{1}\right\}$, then $M_{1}$ and hence $M$ has $\{x, y, z\}$ as a triangle and the theorem follows easily by Theorem 4.1 ; a contradiction. If $E\left(M_{1}\right)=\left\{x, y, z, p_{1}, f\right\}$ for some element $f$, then, as noted above, $\{x, y, z\}$ cannot be a triangle of $M_{1}$. It follows, since $x$ is not in a triad of $M$, that $M_{1}$ is obtained from a 4-element circuit on $\{x, y, z, f\}$ by adding $p_{1}$ in parallel with $x$. Thus $M^{*} \mid\{e, f, y, z\} \simeq U_{2,4}$ and we get a contradiction by applying Lemma 4.3 to $M^{*}$. If $E\left(M_{1}\right)=\left\{p_{1}, f, q_{1}, s\right\}$ where $\left\{q_{1}, s\right\} \subseteq\{x, y, z\}$, then $M_{1}$ is obtained from a triangle on $\left\{q_{1}, f, s\right\}$ by adding $p_{1}$ in parallel with $q_{1}$. Since $x$ is not in a triad of $M, s \neq x$. Now, by Lemma 4.2, both $M \backslash f$ and $M / f$ are nonbinary. In this case, we take $f_{1}=f$ and $f_{2}=e$.

This finishes the proof of the lemma in the case that $p_{1}$ is parallel to some other element of $M_{1}$. From now on, we shall assume that this does not occur. We complete the proof of the lemma by first establishing the existence of the element $f_{1}$. We then extend the argument to obtain $f_{2}$. Now $f_{1}$ certainly exists unless every element of $E\left(M_{1}\right)-\left\{p_{1}, x, y, z\right\}$ is in series with $p_{1}$. In the exceptional case, we consider $M_{1}^{*}$. It has a parallel class $P$ containing $p_{1}$ and has at least two and at most three other elements. Since $M_{1}$ has no 2-circuits, $M_{1}^{*}$ has no 2-cocircuits. Moreover, $M_{1}^{*}$ is connected. Thus $M_{1}^{*}$ has rank 2 and $\{x, y, z\}=E\left(M_{1}^{*}\right)-P$. Hence $\{x, y, z\}$ is a triad of $M_{1}^{*}$ and so $\{x, y, z\}$ is a triangle of $M_{1}$ and hence of $M$. It follows, by Theorem 4.1, that the theorem holds for $M$; a contradiction. This establishes the existence of an element $f_{1}$ with the properties claimed. 


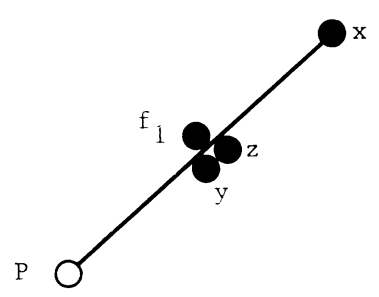

FIGURE 5

To establish the existence of the element $f_{2}$, we shall begin with the assumption that this element does not exist. Thus consider $M \backslash e$ as before and assume that $f_{1}$ is the only element of $E\left(M_{1}\right)-\left\{p_{1}, x, y, z\right\}$ that is not in series with $p_{1}$. As in the case when $p_{1}$ was parallel to one of $x, y$, and $z$, we shall try to choose $f_{2}$ distinct from $e$ and we shall note when this may not be possible.

As before we consider $M_{1}^{*}$. It contains the element $f_{1}$, some subset of $\{x, y, z\}$, and a parallel class $P$ containing $p_{1}$. We know that $M_{1}^{*}$ is connected and has no 2-cocircuits. We look first at the case when all of $x, y$, and $z$ are in $E\left(M_{1}^{*}\right)-$ $P$. Then $\operatorname{rk} M_{1}^{*} \leq 3$. Suppose that $\operatorname{rk} M_{1}^{*}=3$. As $\{x, y, z\}$ is not a triad of $M_{1}^{*}$, the complement of the line of $M_{1}^{*}$ containing $P$ and $f_{1}$ is a cocircuit of $M_{1}^{*}$ properly contained in $\{x, y, z\}$; a contradiction. Thus rk $M_{1}^{*}=2$. As $M_{1}^{*}$ has no 2-circuit containing $x$ and no $U_{2,4}$-minor, $M_{1}^{*}$ is as shown in Figure 5 . But then $M^{*} \mid\left\{e, f_{1}, y, z\right\} \simeq U_{2,4}$ and we get a contradiction by Lemma 4.3.

We conclude that $E\left(M_{1}^{*}\right)-P \nsupseteq\{x, y, z\}$. If $E\left(M_{1}^{*}\right)-P$ contains only one element of $\{x, y, z\}$, then $f_{2}$ exists or else we get the contradiction that $M_{1}^{*}$ has a 2-cocircuit. Thus $E\left(M_{1}^{*}\right)-P$ contains $f_{1}$ together with exactly two elements, say $g$ and $h$, of $\{x, y, z\}$. Moreover, $M_{1}^{*}$ has rank 2 and is binary. Hence $M_{1}^{*}$ is a line having three points, $P,\{a, b\}$, and $\{c\}$, where $\{a, b, c\}=\left\{f_{1}, g, h\right\}$. Therefore $\{e, a, b\}$ is a triad of $M$ and so $x \notin\{a, b\}$. Now look at $M \backslash f_{1}$. If it is a series extension of a 3-connected matroid, then, by Lemma 4.4, $\left\{f_{1}, y, z\right\}$ is a triad of $M$. But $\{e, a, b\}$ is one of $\left\{e, f_{1}, y\right\},\left\{e, f_{1}, z\right\}$, or $\{e, y, z\}$. Thus $M^{*} \mid\left\{e, f_{1}, y, z\right\} \simeq U_{2,4}$ and we get a contradiction by Lemma 4.3. Hence $M \backslash f_{1}$ is not a series extension of a 3-connected matroid. Now apply the first part of the proof of this lemma using $M \backslash f_{1}$ in place of $M \backslash e$ to obtain an element $f_{2}$ not in $\left\{f_{1}, x, y, z\right\}$ for which both $M \backslash f_{2}$ and $M / f_{2}$ are nonbinary. We observe that possibly $f_{2}=e$ but, in that case, the structure of $M_{1}^{*}$ is as indicated above. This completes the proof of Lemma 4.7.

(4.10) LEMMA. $M$ has two distinct elements $f_{1}$ and $f_{2}$ that are not in $\{x, y, z\}$ such that all of $M \backslash f_{1}, M / f_{1}, M \backslash f_{2}$, and $M / f_{2}$ are nonbinary.

Proof. By Theorem 4.1, $M^{*}$ has a $U_{2,4}$-minor using $\{y, z\}$. Therefore, as $\operatorname{rk} M^{*} \geq 4, M^{*}$ has an element $e$ not in $\{x, y, z\}$ such that $M^{*} / e$ and hence $M \backslash e$ is nonbinary. If $M \backslash e$ is not a series extension of a 3 -connected matroid, then this lemma is immediate from Lemma 4.7. Thus we may assume that $M \backslash e$ is a series extension of a 3-connected matroid. In that case, by Lemma $4.4,\{e, y, z\}$ is a triad of $M$ and so $\{e, y, z\}$ is a triangle of $M^{*}$. By Theorem 4.1, it follows that $M^{*}$ has a $U_{2,4}$-minor using $\{e, y, z\}$, hence, as $\operatorname{rk} M^{*} \geq 4, M^{*}$ has an element $f$ not in $\{e, x, y, z\}$ such that $M^{*} / f$ is nonbinary. Thus $M \backslash f$ is nonbinary. If $M \backslash f$ is not a series extension of a 3-connected matroid, the lemma is immediate from Lemma 4.7. If $M \backslash f$ is a series extension of a 3 -connected matroid, then $\{f, y, z\}$ is a triad 


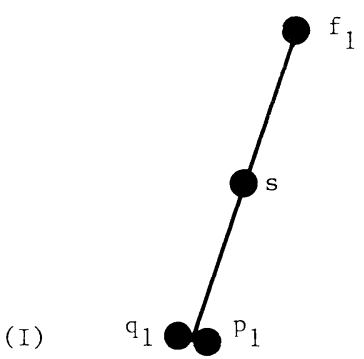

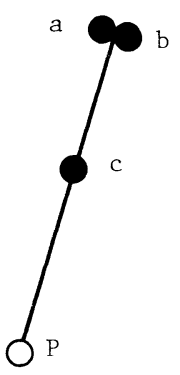

FIGURE 6

of $M$ and so $M^{*} \mid\{e, f, y, z\} \simeq U_{2,4}$. We now obtain a contradiction by applying Lemma 4.3 to $M^{*}$.

On combining the next result with the preceding lemma, Theorem 1.9 will follow.

(4.11) LEMMA. $E(M)-\{x, y, z\}$ does not contain two distinct elements $f_{1}$ and $f_{2}$ such that all of $M \backslash f_{1}, M / f_{1}, M \backslash f_{2}$, and $M / f_{2}$ are nonbinary.

Proof. We assume that the elements $f_{1}$ and $f_{2}$ do exist. By Lemma 4.5 and Theorem 1.6, both $M / f_{1}$ and $M / f_{2}$ are parallel extensions of 3-connected matroids, and neither $M \backslash f_{1}$ nor $M \backslash f_{2}$ is a series extension of a 3-connected matroid. Moreover, by Lemma 4.3 and the dual of the argument in Lemma 4.4, two of $x, y$, and $z$ form a triangle with $f_{1}$, and a different pair forms a triangle with $f_{2}$. Now $x$ has been distinguished in $\{x, y, z\}$ by the assumption that $M \backslash x$ is nonbinary and 3 -eonnected. It follows then that, without loss of generality, we may assume that $\left\{f_{1}, x, y\right\}$ is a triangle of $M$ and that either (i) $\left\{f_{2}, x, z\right\}$ or (ii) $\left\{f_{2}, y, z\right\}$ is also a triangle of $M$. In both cases, if $M$ has an element $f_{3}$ that is not in $\left\{f_{1}, f_{2}, x, y, z\right\}$ such that both $M \backslash f_{3}$ and $M / f_{3}$ are nonbinary, then we obtain a contradiction to Lemma 4.6. A contradiction is also obtained if $\left\{f_{1}, x, y, z\right\}$ contains a triangle other than $\left\{f_{1}, x, y\right\}$. Now consider $M \backslash f_{2}$. It is nonbinary and is not a series extension of a 3 -connected matroid. Thus $M \backslash f_{2}=P\left(\left(M_{1}, p_{1}\right),\left(M_{2}, p_{2}\right)\right) \backslash p$ where $M_{2}$ is nonbinary, $M_{1}$ is not a single circuit, and $\left|E\left(M_{1}\right)\right| \geq 4$. We choose $M_{1}$ here so that $\left|E\left(M_{1}\right)\right|$ is maximum subject to these conditions. Now $f_{1} \in E\left(M_{1}\right)$, otherwise the argument of Lemma 4.7 establishes the existence of the required element $f_{3}$. From the proof of that lemma, it follows that the element $f_{3}$ exists unless either $M_{1}$ is as shown in Figure 6(I) where $\left\{q_{1}, s\right\} \subseteq\{x, y, z\}$ and $s \neq x$; or $E\left(M_{1}^{*}\right)-P$ contains $f_{1}$ and exactly two elements of $\{x, y, z\}$ and $M_{1}^{*}$ is as shown in Figure 6(II) where $\{a, b, c\}=\left\{f_{1}, g, h\right\},\{g, h\} \subseteq\{x, y, z\}$, and $x \notin\{a, b\}$. In the former case, $\left\{f_{1}, q_{1}, s\right\}$ is a triangle of $M$ contained in $\left\{f_{1}, x, y, z\right\}$. Thus $\left\{q_{1}, s\right\}=\{x, y\}$. But $s \neq x$, so $q_{1}=x$ and $s=y$. Therefore, in case $(\mathrm{I}),\left\{f_{1}, f_{2}, y\right\}$ is a triad of $M$. This is also true in case (II) for, in that case, $\{a, b, c\}$ is a triad of $M_{1}^{*}$ and hence is a triangle of $M$. Since it is contained in $\left\{f_{1}, x, y, z\right\}$, it must be $\left\{f_{1}, x, y\right\}$. Now $x \notin\{a, b\}$, so $\{a, b\}=\left\{f_{1}, y\right\}$. Hence we do indeed have that in both cases (I) and (II), $\left\{f_{1}, y, f_{2}\right\}$ is a triad of $M$. Therefore if $\left\{f_{2}, x, z\right\}$ is a triangle of $M$ it meets this triad in a single element. This contradiction eliminates case (i) above. Hence we need only consider case (ii). It follows that in both cases (I) and (II) we may assume that $M_{1}$ is obtained from the cycle matroid of the graph shown in Figure 7 by adding a (possibly empty) set of edges in series with $p_{1}$. 


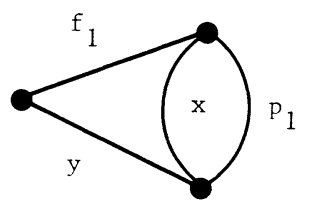

\section{FIGURE 7}

If the series class of $M_{1}$ containing $p_{1}$ contains elements $u_{1}$ and $v_{1}$ distinct from $p_{1}$, then $\left\{f_{2}, u_{1}, v_{1}\right\}$ is a triad of $M$. As $z \in E\left(M_{2}\right)$, this triad meets the triangle $\left\{f_{2}, y, z\right\}$ in a single element; a contradiction. Thus either

(1) $M_{1}$ is as shown in Figure 7; or

(2) $M_{1}$ has one extra element $u_{1}$ in series with $p_{1}$.

In case (2), $\left\{f_{1}, x, u_{1}\right\}$ and $\left\{x, y, u_{1}\right\}$ are triads of $M_{1}$ and hence of $M \backslash f_{2}$. Since $x$ is not in a triad of $M$, neither $\left\{f_{1}, x, u_{1}\right\}$ nor $\left\{x, y, u_{1}\right\}$ is a cocircuit of $M$ and therefore both $\left\{f_{2}, f_{1}, x, u_{1}\right\}$ and $\left\{f_{2}, x, y, u_{1}\right\}$ are cocircuits of $M$. Thus $M \backslash u_{1}$ has $\left\{f_{1}, f_{2}, x\right\}$ and $\left\{f_{2}, x, y\right\}$ as cocircuits. Therefore $\left(M \backslash u_{1}\right)^{*} \mid\left\{x, y, f_{1}, f_{2}\right\}$ is either a 4-point line or a line having three points, $\{x\},\left\{y, f_{1}\right\},\left\{f_{2}\right\}$. In the first case, $\left(M \backslash u_{1}\right)^{*}$ is nonbinary, so $M \backslash u_{1}$ is also nonbinary. Since $M / u_{1}$ has an $M_{2}$-minor, it too is nonbinary. Thus we can take $f_{3}=u_{1}$ and the required result follows. In the second case, $\left\{y, f_{1}\right\}$ is a cocircuit of $M \backslash u_{1}$. Thus $\left\{u_{1}, y, f_{1}\right\}$ is a triad of $M$ and hence of $M_{1}$. But $\left\{x, p_{1}, u_{1}\right\}$ is a triangle of $M_{1}$ meeting this triad in a single element; a contradiction. Thus case (2) cannot occur.

Now consider case (1). Because $x$ is parallel to $p_{1}$ in $M_{1}$, the matroid $M \backslash f_{1}, f_{2}, y$ is obtained from $M_{2}$ simply by relabelling the element $p_{2}$ by $x$. For the rest of the proof we shall identify $M_{2}$ with $M \backslash f_{1}, f_{2}, y$.

We shall show next that $M_{2}$ has a $U_{2,4}$-minor using $\{x, z\}$. If $M_{2}$ is 3-connected this is immediate from Theorem 4.1. If $M_{2}$ is not 3 -connected, then, by Theorem $1.2, M_{2}$ is the 2-sum of matroids $M_{2,1}$ and $M_{2,2}$ with respect to the basepoints $s_{1}$ and $s_{2}$ where $x \in E\left(M_{2,2}\right)$ and $\left|E\left(M_{2,1}\right)\right|$ is maximum subject to these conditions. If $M_{2,2}$ is not 3 -connected, then it is the 2-sum of matroids $M_{2,3}$ and $M_{2,4}$ with respect to the basepoints $s_{3}$ and $s_{4}$ where $x \in E\left(M_{2,4}\right)$ and $\left|E\left(M_{2,3}\right)\right|$ is maximum. By the choice of $M_{2,1}$, we must have that $s_{2} \in E\left(M_{2,4}\right)$. If $M_{2,4}$ is not 3-connected, then it is the 2-sum of matroids $M_{2,5}$ and $M_{2,6}$ with respect to the basepoints $s_{5}$ and $s_{6}$ where $x \in E\left(M_{2,6}\right)$ and $\left|E\left(M_{2,5}\right)\right|$ is maximum. Again the choice of $M_{2,1}$ and $M_{2,3}$ guarantees that $s_{2}$ and $s_{4}$ are in $E\left(M_{2,6}\right)$. Repeating this process, we eventually obtain a 3 -connected matroid $M_{2,2 k}$ containing $x, s_{2}, s_{4}, \ldots, s_{2 k}$ such that $M_{2}$ is formed from $M_{2,2 k}$ by taking the 2 -sum of $M_{2,2 k}$ and $M_{2,1}$ with respect to $s_{2}$ and $s_{1}$; then taking the 2-sum of the result and $M_{2,3}$ with respect to $s_{4}$ and $s_{3}$ and so on. Evidently, for all $j$ in $\{1,2, \ldots, k\}, M_{2}$ is the 2 -sum of $M_{2,2 j-1}$ and another matroid, say $M_{2,2 j-1}^{\prime}$, which contains $x$. As $M_{2}$ is nonbinary, at least one of the parts of this 2-sum is nonbinary. If $M_{2,2 j-1}$ is nonbinary, then $M \backslash f_{2}$ is the 2-sum of $M_{2,2 j-1}$ and another matroid $M_{1}^{\prime}$, which is the 2-sum of $M_{1}$ and $M_{2,2 j-1}^{\prime}$. But $M_{1}^{\prime}$ has more elements than $M_{1}$; a contradiction to the choice of $M_{1}$. It follows that $M_{2,2 j-1}$ is binary. Since this is true for all $j$ in $\{1,2, \ldots, k\}$, we must have that $M_{2,2 k}$ is nonbinary. It follows, by Theorem 4.1 (or see $[8,(4.2)]$ ), that, whether it is 3-connected or not, $M_{2}$ has a $U_{2,4}$-minor using $\{x, z\}$. Suppose that this minor is $M_{2} / A \backslash B$. then $M \backslash f_{2} / A \backslash B$ is the matroid shown in Figure 8 . 


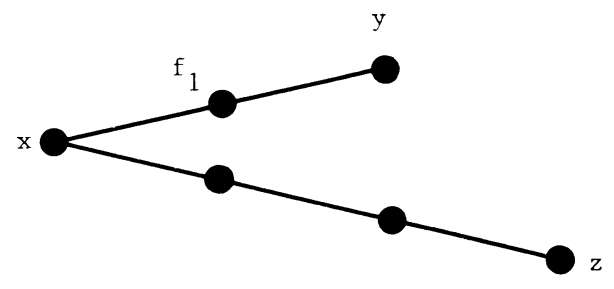

FIGURE 8

Now, we may assume that $A$ is independent in $M_{2}$. As $A \cap\left\{y, f_{1}, f_{2}\right\}$ is empty and $\left\{y, f_{1}, f_{2}\right\}$ is a cocircuit of $M$, both $A \cup f_{2}$ and $A \cup y$ are independent in $M$. We recall that $\left\{f_{2}, y, z\right\}$ is a circuit of $M$. Thus either $\left\{f_{2}, y, z\right\}$ is a circuit of $M / A$ or $\left\{f_{2}, y, z\right\}$ properly contains a circuit of $M / A$. In the first case, we are forced to get a $W^{3}$-minor of $M$ having $\{x, y, z\}$ as its set of spokes; a contradiction. In the second case, since every circuit of $M$ containing $f_{2}$ must meet both $E\left(M_{1}\right)$ and $E\left(M_{2}\right)$, and $\{y, z\}$ is independent in $M / A$, we must have that $\left\{f_{2}, y\right\}$ is a circuit of $M / A$. Thus $A \cup\left\{f_{2}, y\right\}$ contains a circuit $C$ of $M$. Moreover, $\left\{f_{2}, y\right\} \subseteq C$ since both $A \cup f_{2}$ and $A \cup y$ are independent. But $\left\{f_{2}, y, z\right\}$ is also a circuit of $M$ and so, by exchange, $M$ has a circuit containing $z$ and contained in $A \cup\left\{f_{2}, z\right\}$. The element $f_{2}$ is not in this circuit, otherwise the exact 2-separation we have of $M \backslash f_{2}$ extends to an exact 2 -separation of the 3 -connected matroid $M$; a contradiction. But now in $M / A$, the element $z$ is a loop; a contradiction. This completes the proof of the lemma, thereby finishing the proof of the theorem.

ACKNOWLEDGMENT. The author thanks the referee for several suggested improvements to this paper.

\section{REFERENCES}

1. R. E. Bixby, l-matrices and a characterization of binary matroids, Discrete Math. 8 (1974), 139-145.

2. A simple theorem on 3-connectivity, Linear Algebra Appl. 45 (1982), 123-126.

3. T. Brylawski, A combinatorial model for series-parallel networks, Trans. Amer. Math. Soc. 154 (1971), 1-22.

4. J. Kahn, A problem of P. Seymour on nonbinary matroids, Combinatorica 5 (1985), 319323.

5. J. G. Oxley, On matroid connectivity, Quart. J. Math. Oxford (2) 32 (1981), 193-208.

6. - On the intersections of circuits and cocircuits in matroids, Combinatorica 4 (1984), 187-195.

7. P. D. Seymour, Decomposition of regular matroids, J. Combin. Theory Ser. B 28 (1980), 305-359.

8. __ On minors of non-binary matroids, Combinatorica 1 (1981), 387-394.

9. - Minors of 3-connected matroids, Eurpoean J. Combin. 7 (1986), 171-176.

10. , Triples in matroid circuits, European J. Combin. 7 (1986), 177-185.

11. W. T. Tutte, Connectivity in matroids, Canad. J. Math. 18 (1966), 1301-1324.

12. D. J. A. Welsh, Matroid theory, Academic Press, London, 1976.

Department of Mathematics, Louisiana State University, Baton Rouge, LOUISIANA 70803 